\title{
First-line therapy, autologous stem-cell transplantation, and post-transplantation maintenance in the management of newly diagnosed mantle cell lymphoma
}

\author{
S. Bhella MD, ${ }^{*}$ N.P. Varela $\mathrm{PhD}_{,}^{\dagger}$ A. Aw MD, ${ }^{\ddagger}$ C. Bredeson MD MSc, ${ }^{\S}$ M. Cheung MD MSc, \\ M. Crump MD, ${ }^{*}$ G. Fraser MD, ${ }^{\#}$ S. Sajkowski, ${ }^{* *}$ and T. Kouroukis MD MSc ${ }^{\#}$
}

\begin{abstract}
Background In Ontario, no clearly defined standard of care for the management of mantle cell lymphoma (MCL) has been developed, and substantial variability from centre to centre is evident. This guidance document was prompted by the need to harmonize practice in Ontario with respect to first-line, conditioning, and post-transplantation maintenance therapy for patients newly diagnosed with transplantation-eligible MCL.

Methods The MEDLINE and EMBASE databases were systematically searched from January 2013 to January 2020 for evidence, and the best available evidence was used to draft recommendations relevant to first-line therapy, autologous stem-cell transplantation, and post-transplantation maintenance in the management of transplantation-eligible newly diagnosed MCL. Final approval of this guidance document was obtained from the Stem Cell Transplant Advisory Committee.
\end{abstract}

Recommendations These recommendations apply to all cases of transplantation-eligible newly diagnosed MCL:

Alternating cycles of R-CHOP (rituximab plus cyclophosphamide-doxorubicin-vincristine-prednisolone) and R-DHAP [rituximab plus dexamethasone-high-dose cytarabine-cisplatin] is the recommended first-line treatment for symptomatic patients newly diagnosed with MCL before autologous stem-cell transplantation (ASCT). Rituximab plus hyperfractionated cyclophosphamide-vincristine-doxorubicin-dexamethasone (R-hyperCVAD), alternating with methotrexate and cytarabine, is not recommended for the treatment of patients with newly diagnosed MCL.

BEAM (carmustine-etoposide-cytarabine-melphalan), BEAC (carmustine-etoposide-cytarabine-cyclophosphamide), and total-body irradiation-based regimens are reasonable conditioning options for patients with MCL who have responded to first-line therapy and who are undergoing ASCT.

Maintenance therapy with rituximab is recommended for patients with newly diagnosed MCL who have undergone ASCT.

Key Words Mantle cell lymphoma, first-line therapy, autologous stem-cell transplantation, maintenance therapy

\section{INTRODUCTION}

In Ontario, there is no clearly defined standard of care for patients newly diagnosed with transplantation-eligible mantle cell lymphoma (MCL). A variety of first-line chemoimmunotherapy induction and consolidative approaches to autologous stem-cell transplantation (ASCT) are used in young, fit patients. Induction treatments during upfront management before transplantation have included rituximab-bendamustine, R-hypercvad (rituximab plus hyperfractionated cyclophosphamide-vincristinedoxorubicin-dexamethasone), and R-CHOP (rituximab plus 
cyclophosphamide-doxorubicin-vincristine-prednisolone) alternating with R-DHAP (rituximab plus dexamethasonehigh-dose cytarabine-cisplatin). Conditioning regimens can vary, and practice in Ontario is guided by retrospective data supporting BEAM (carmustine-etoposide-cytarabinemelphalan), BEAC (carmustine-etoposide-cytarabinecyclophosphamide), and total-body irradiation (ТВI) approaches ${ }^{1}$. The evidence with respect to consolidative ASCT has demonstrated a progression-free survival (PFS) benefit in eligible patients who underwent $\mathrm{ASCT}^{2}$.

In MCL, ASCT was established as the standard of care based on evidence from the European MCL Network Tri$\mathrm{al}^{3}$, which demonstrated improved PFs, reaching a median of 39 months in patients who underwent ASCT compared with 17 months in patients who received interferon alfa instead of transplantation. To improve outcomes and prolong remission, consolidation with ASCT is frequently used in most patients with newly diagnosed symptomatic transplantation-eligible MCL. Decisions about eligibility for ASCT should be made on a case-by-case basis, considering factors such as age, disease stage (III, IV), functional status, and organ function; when considering ASCT, patients should also be involved to discuss the potential benefits and risks of the proposed treatment and treatment alternatives, if any.

The objective of this article was to provide guidance based on the available evidence with respect to best practices for first-line therapy, conditioning regimens, timing of ASCT, and maintenance therapy for patients newly diagnosed with transplantation-eligible MCL. Based on that objective, the authors derived these research questions:

1. For patients newly diagnosed with transplantationeligible MCL, what is the preferred induction regimen?

2. For patients who achieve a partial or better response to induction therapy, does the addition of ASCT lead to longer and better PFS or overall survival (OS), or both, when compared with no ASCT? If so, what is the preferred conditioning regimen, and what is the most appropriate timing for mobilization before ASCT (ideal number of induction chemotherapy cycles)?

3. For patients with MCL in remission after ASCT, does the addition of rituximab or interferon alfa maintenance therapy lead to longer and better PFS or os?

\section{METHODS}

A guideline published by the U.K. National Institute for Health and Care Excellence ${ }^{4}$ was used as a source of reference rather than as the main evidence source for the present guidance document, because current evidence from phase II and III randomized controlled trials (RCTs) and large prospective observational studies might lead to a change in some of the recommendations. Our evidence review was conducted in two planned stages, including a search for systematic reviews and a primary literature review.

\section{Search for Existing Systematic Reviews}

The Cochrane Database of Systematic Reviews (https:// www.cochranelibrary.com/cdsr/table-of-contents), together with the electronic databases MEDLINE (Ovid) and EMBASE (Ovid) were searched for relevant publications dated from January 2013 to January 2019. Systematic reviews more than 6 years in the past were considered not relevant, because the main goal of the search was to identify recent secondary sources covering the primary literature that might be helpful in developing the recommendations. The full search strategy is available in supplemental Appendix 1.

\section{Search for Primary Literature}

In the absence of systematic reviews addressing the research questions, other methods were used to search the primary literature. The electronic databases MEDLINE (Ovid) and EMBASE (Ovid) were searched for relevant articles from the completion date of the search conducted by the U.K. National Institute for Health and Care Excellence for its 2016 guideline (2015) to January 2019 for research questions 1 and 2, and from 1996 to 2019 for question 3. The literature search was updated in January 2020. The search strategy included a logical combination of terms for the condition (MCL) and the interventions (systemic therapy, ASCT, maintenance, rituximab, interferon alfa). The full literature search strategy used to retrieve potential primary studies is available in supplemental Appendix 1.

\section{Study Selection Criteria and Process}

Studies were required to be phase II and III RCTs evaluating any of the following indications in the management of patients newly diagnosed with ASCT-eligible MCL: first-line therapy, conditioning regimens, timing to ASCT, and maintenance after ASCT. If no randomized evidence was available, primary observational studies fully published in English were considered if they were prospective comparative studies with at least 25 participants per treatment group or single-arm studies with at least 100 participants for question 1 (first-line therapy) and 50 participants for question 2 (ASCT). No single-arm studies were considered for question 3 (maintenance). Studies had to have reported at least 1 of the following outcomes by treatment group: OS, PFS, quality of life, and toxicities. Retrospective studies, letters, case reports, comments, books, notes, and editorials that reported clinical trial outcomes were excluded. Titles and abstracts that resulted from the search were reviewed by a single author (NPV). Items that warranted a full-text review were reviewed by NPV in collaboration with the other authors.

\section{Data Extraction and Assessment of Study Quality and Potential Bias}

All included primary studies underwent data extraction by one author (NPV), with all extracted data and information subsequently being audited by an independent auditor. Risk of bias for each included RCT was assessed using the Cochrane Collaboration's Risk of Bias assessment tool, focusing on the randomization process, allocation concealment, blinding, data availability, and outcome measurement ${ }^{5}$. Single-arm evidence was assessed based on full reporting of the patient selection criteria, the intervention, the follow-up period, and all relevant outcomes, together with the methods used to measure them.

\section{Synthesizing the Evidence}

Clinical heterogeneity between the studies in terms of the interventions and research settings, as well as inconsistent 
reporting of outcomes, prevented a meta-analysis from being performed. Instead, data were synthesized in tables and are described narratively in the text.

\section{RESULTS}

\section{Search for Existing Systematic Reviews}

From among sixteen citations identified in the MEDLINE and EMBASE databases and the Cochrane Database of Systematic Reviews, one systematic review focused on the efficacy of rituximab maintenance therapy in patients with $\mathrm{MCL}^{6}$, but differences in its target population and study eligibility compared with the systematic review in the evidentiary base prevented its inclusion.

\section{Search for Primary Literature}

After removal of duplicates, the initial literature search resulted in 3520 citations, of which 246 were determined to be eligible for full-text review. After that review, five fullreport publications from 4 studies were found to be relevant and were therefore included to inform the recommendations concerning the management of newly diagnosed stem-cell transplantation-eligible MCL. The remaining 241 publications were excluded because they failed to pass the predefined inclusion criteria.

\section{Study Design and Quality}

The five included publications assessed the management of MCL eligible for ASCT and reported 3 of the outcomes of interest: PFS, OS, toxicities. One randomized phase III trial ${ }^{7}$, two publications from randomized multi-institutional phase II trials appraising the same evidence at two time points ${ }^{8,9}$, and one large prospective single-arm trial with a 15 -year follow-up period (the Nordic MCL2 trial) ${ }^{10}$ focused on firstline therapy, including ASCT. Just one randomized phase III trial focused on post-transplantation maintenance ${ }^{11}$. No studies reported on conditioning regimens or number of induction chemotherapy cycles for mobilization before ASCT. None of the identified studies reported on quality of life. Tables I and II show the characteristics and outcomes of the included studies.

\section{OUTCOMES}

\section{Preferred Frontline Induction Therapy for Patients Newly Diagnosed with ASCT-Eligible MCL}

\section{R-CHOP: Alternating R-CHOP/R-DHAP Plus Cytarabine Compared with R-CHOP Alone}

First-Line Treatment: One randomized open-label phase III trial by the European MCL Network ${ }^{7}$ demonstrated that, in 466 patients 65 years of age or younger, alternating course of $3 \times$ R-CHOP and $3 \times$ R-DHAP followed by high-dose cytarabine, compared with R-CHOP without cytarabine, resulted in a significantly longer time to treatment failure that was also observed across MCL prognostic index (age, performance status, S-lactate dehydrogenase, and white blood cell count) risk groups (median: 9.1 years vs. 3.9 years; 5 -year rate: $65 \%$ vs. $40 \%$; HR: 0.56 ; $p=0.038$; Table II). To avoid second-line treatment (ASCT) interaction with the primary analysis of first-line therapy, time to treatment failure (from randomization to stable disease after at least 4 induction cycles, progression, or death from any cause) was used as the primary outcome rather than PFS. Significant hematologic (grades 3-4) and renal toxicities (grades 1-2) were more common in patients treated with the cytarabine-containing regimen, but the authors reported that those toxicities were not associated with excess mortality and did not prevent subsequent ASCT; the same proportion of patients underwent stem-cell transplantation in both groups $(84 \%$ in the R-CHOP/R-DHAP + cytarabine regimen and $85 \%$ in the $\mathrm{R}-\mathrm{CHOP}$ regimen).

Conditioning Regimen: Toxicities for both conditioning regimes (ТВI + cytarabine-melphalan vs. TBI + high-dose cyclophosphamide) were similar, except for increased liver toxicity with the $\mathrm{R}$-CHOP regimen [transaminases (grades 1-2) and constipation].

Post-ASCT: After stem-cell transplantation, a statistically significant improvement in PFS was observed for patients treated with the R-CHOP/R-DHAP plus cytarabine regimen compared with those treated with the R-CHOP regimen [PFS, median from randomization: 9.1 years vs. 4.3 years; 5 -year rate: $65 \%$ vs. $44 \%$; HR: 0.55 ; $95 \%$ CI: 0.42 to $0.71 ; p<0.0001$; PFS, median from ASCT: not reached (NR) vs. 4.5 years; 5 -year rate: $73 \%$ vs. $45 \%$; HR: 0.45 ; $95 \%$ CI: 0.33 to $0.63 ; p<0.0001$ ], but no significant difference in os was observed between the two regimens (median: 9.8 years vs. NR; 5 -year rate: $76 \%$ vs. $69 \%$; HR: 0.78 ; $95 \%$ CI: 0.57 to $1.07 ; p=0.12$ ). The proportion of ASCT-related deaths in remission was reported to be the same in both groups (3.4\%).

The quality of the evidence in the European MCL Network trial is considered high: subjects were adequately randomized, resulting in comparable study groups; participants were treated according to intended interventions and were followed for an extensive period of time, with few lost to follow-up; and data were analyzed in accordance with a pre-specified plan (see supplementary Appendix 2 for details).

\section{Rituximab-Bendamustine Compared with R-HyperCVAD}

A randomized phase II trial (S1106) comparing R-hyperCVAD (RH) with rituximab-bendamustine (RB) at two time points ${ }^{8,9}$ provided very low-quality evidence against $\mathrm{RH}$ as a feasible induction regimen before ASCT because of an unacceptable high mobilization failure rate $(29 \%)$, which prompted the premature closure of the study (Table II). Although the 2- and 5-year PFS and os showed no significant differences between patients treated with RB and those treated with $\mathrm{RH}$, the $\mathrm{RH}$ regimen was more toxic and was associated with a higher rate of stem-cell mobilization failure. Only 53 of a planned 160 patients were accrued $(\mathrm{RH}$, 18 ; $\mathrm{RB}, 35$ ), and the small sample size therefore limited the precision of the estimates because the true significance of the data could not be assessed.

Evidence from the S1106 trial is considered to be of very low quality because the data were not analyzed in accordance with the pre-specified plan, and an unacceptably high mobilization failure rate on one arm of the study (RH) prompted premature study closure. 
GUIDANCE FOR THE MANAGEMENT OF MANTLE CELL LYMPHOMA, Bhella et al.

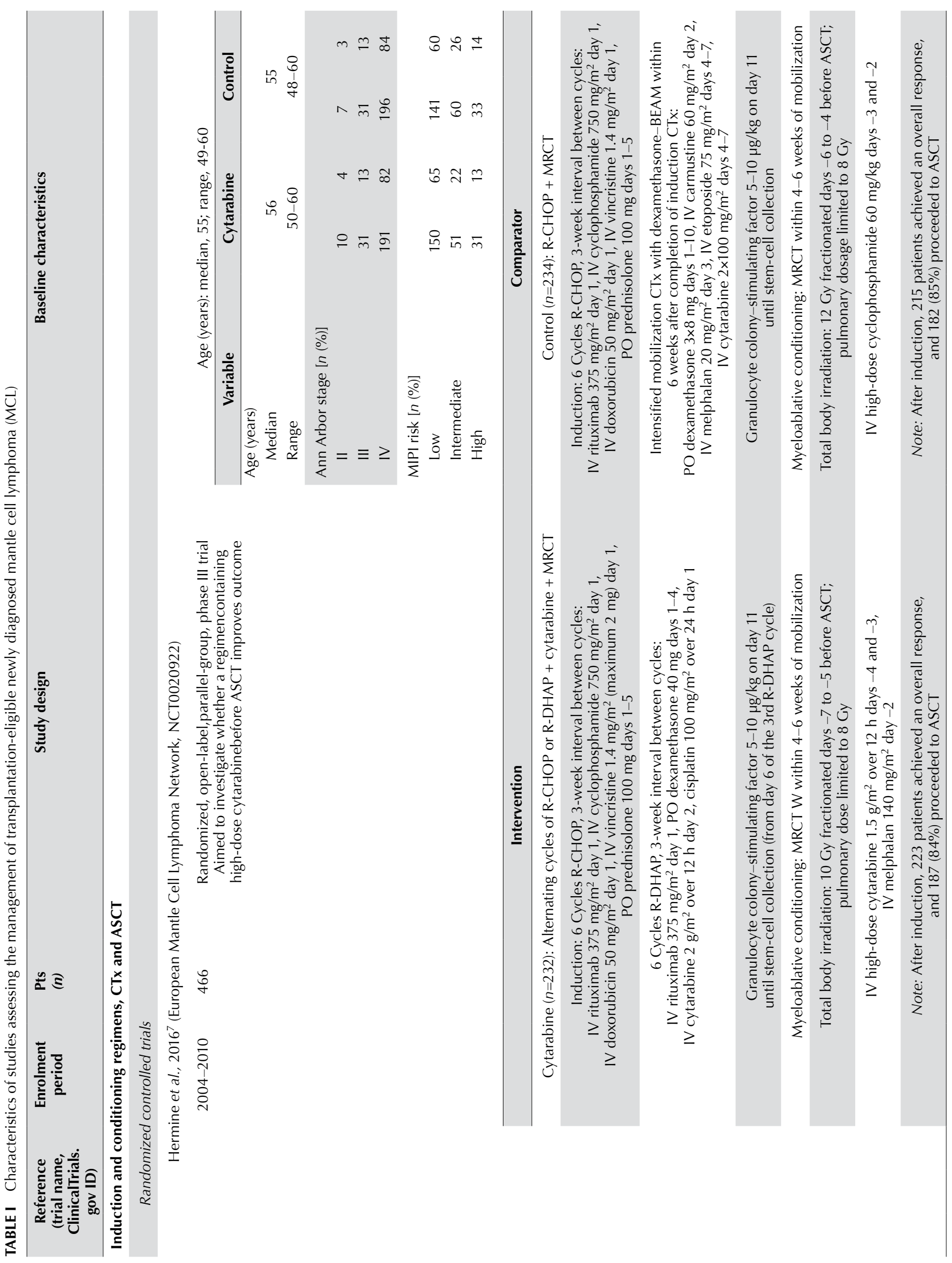




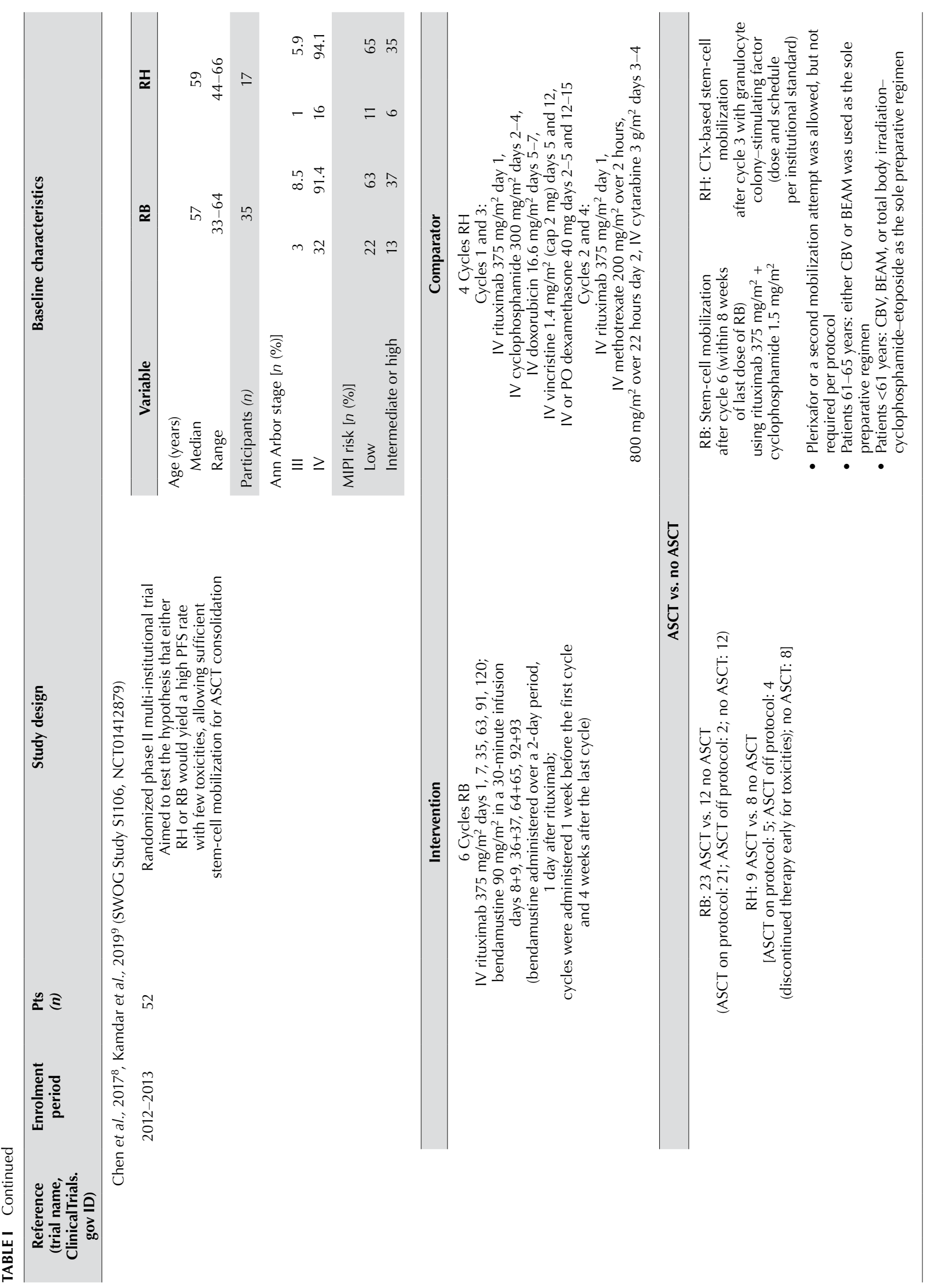


GUIDANCE FOR THE MANAGEMENT OF MANTLE CELL LYMPHOMA, Bhella et al.

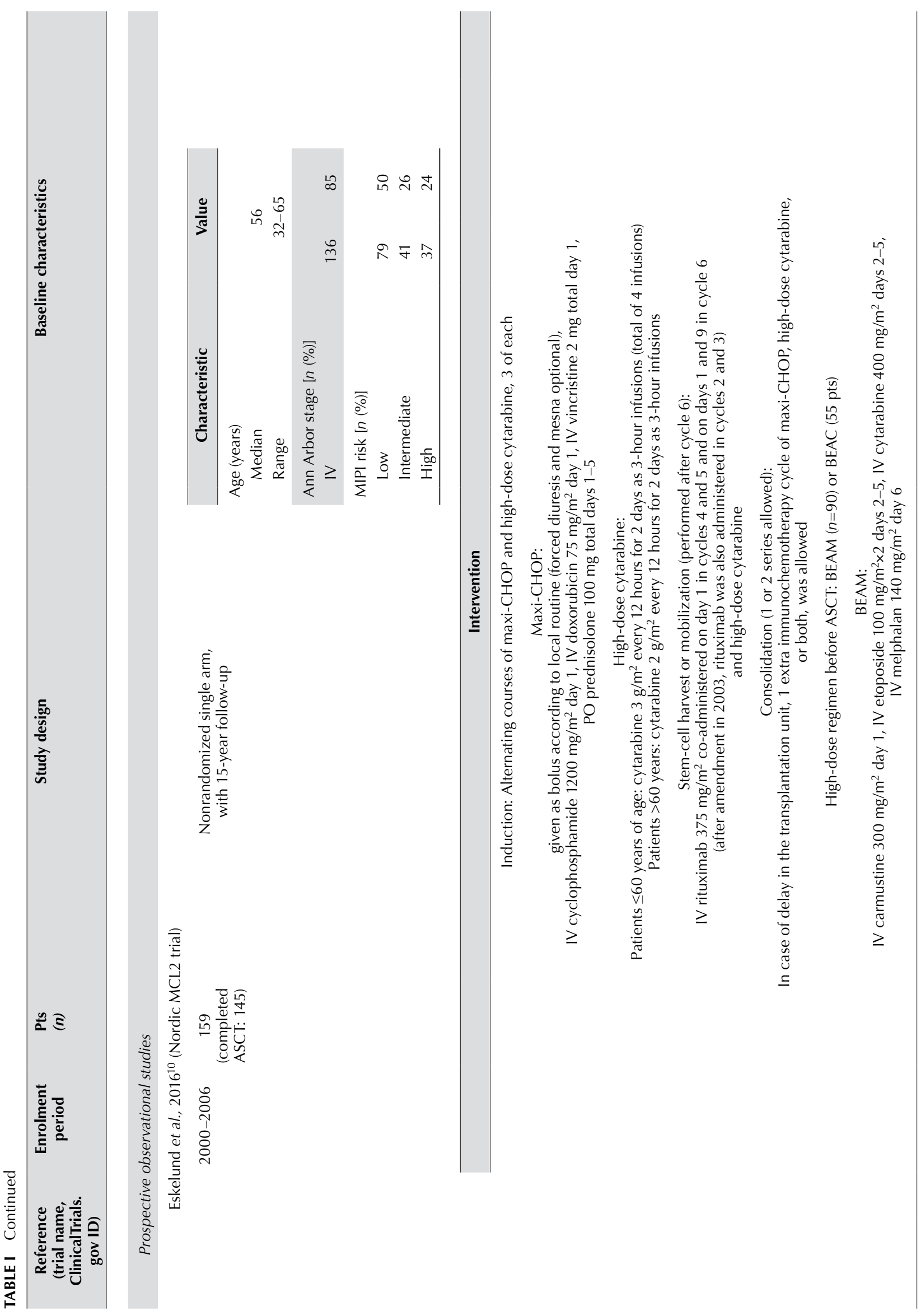




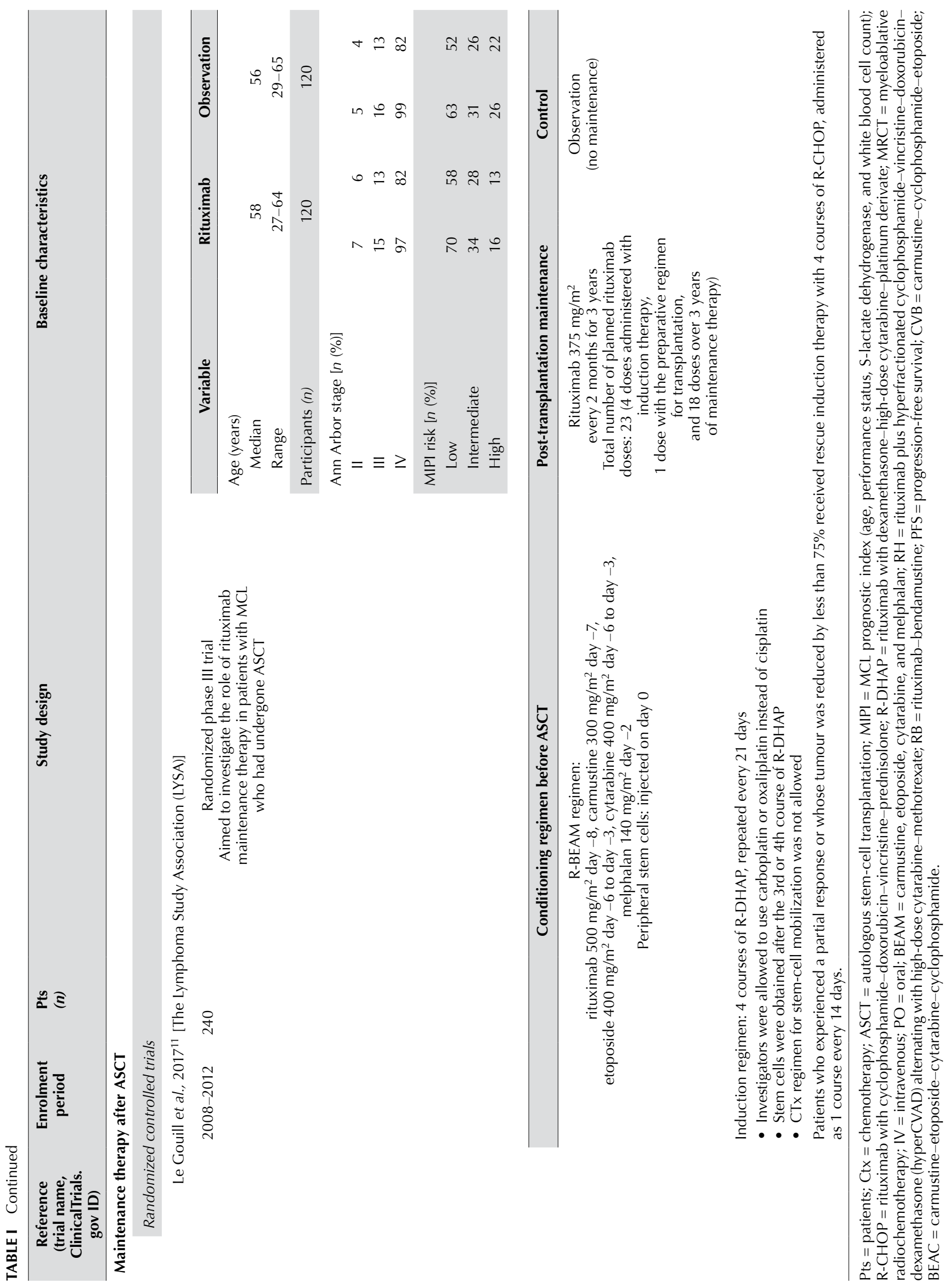




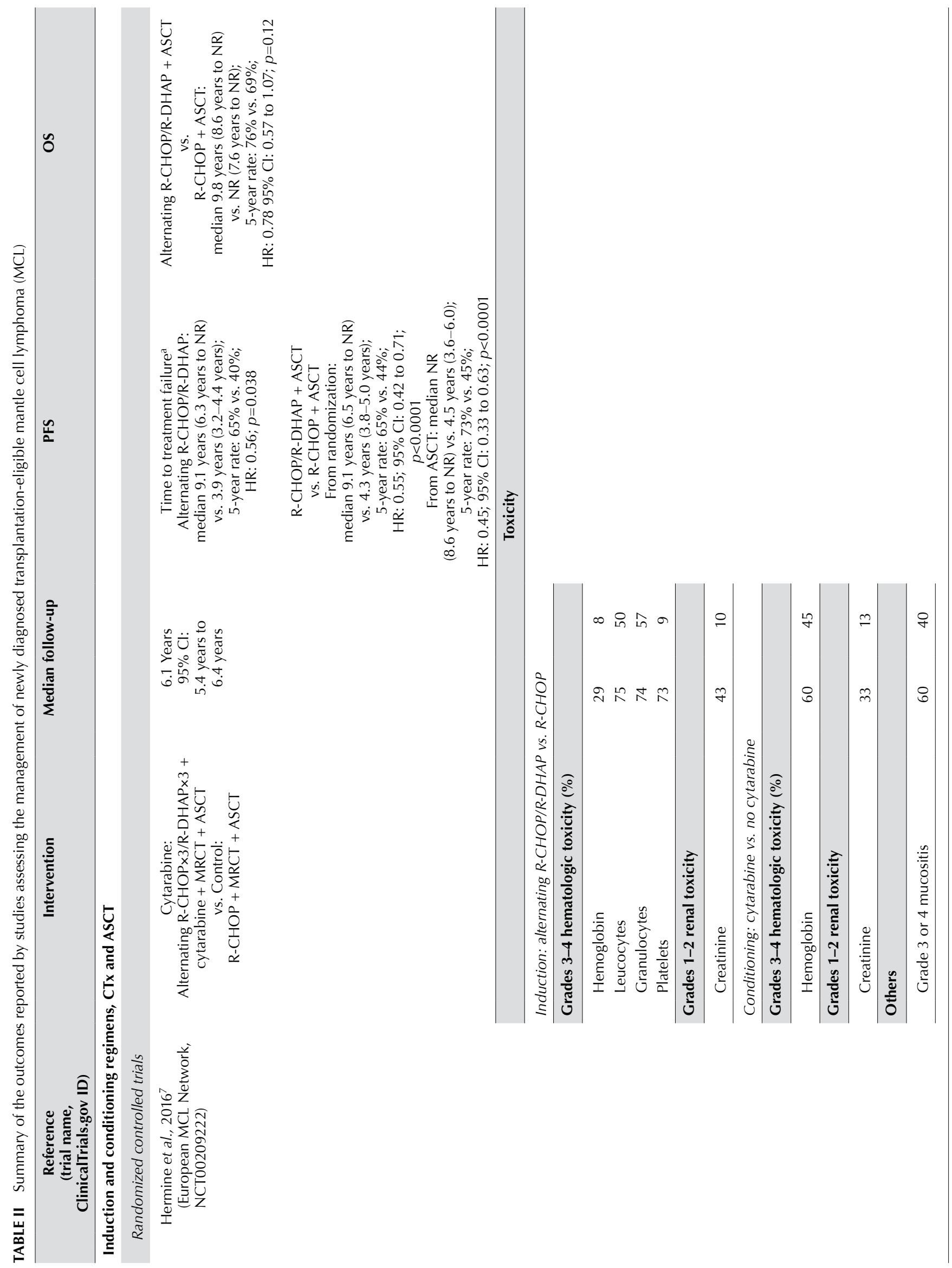




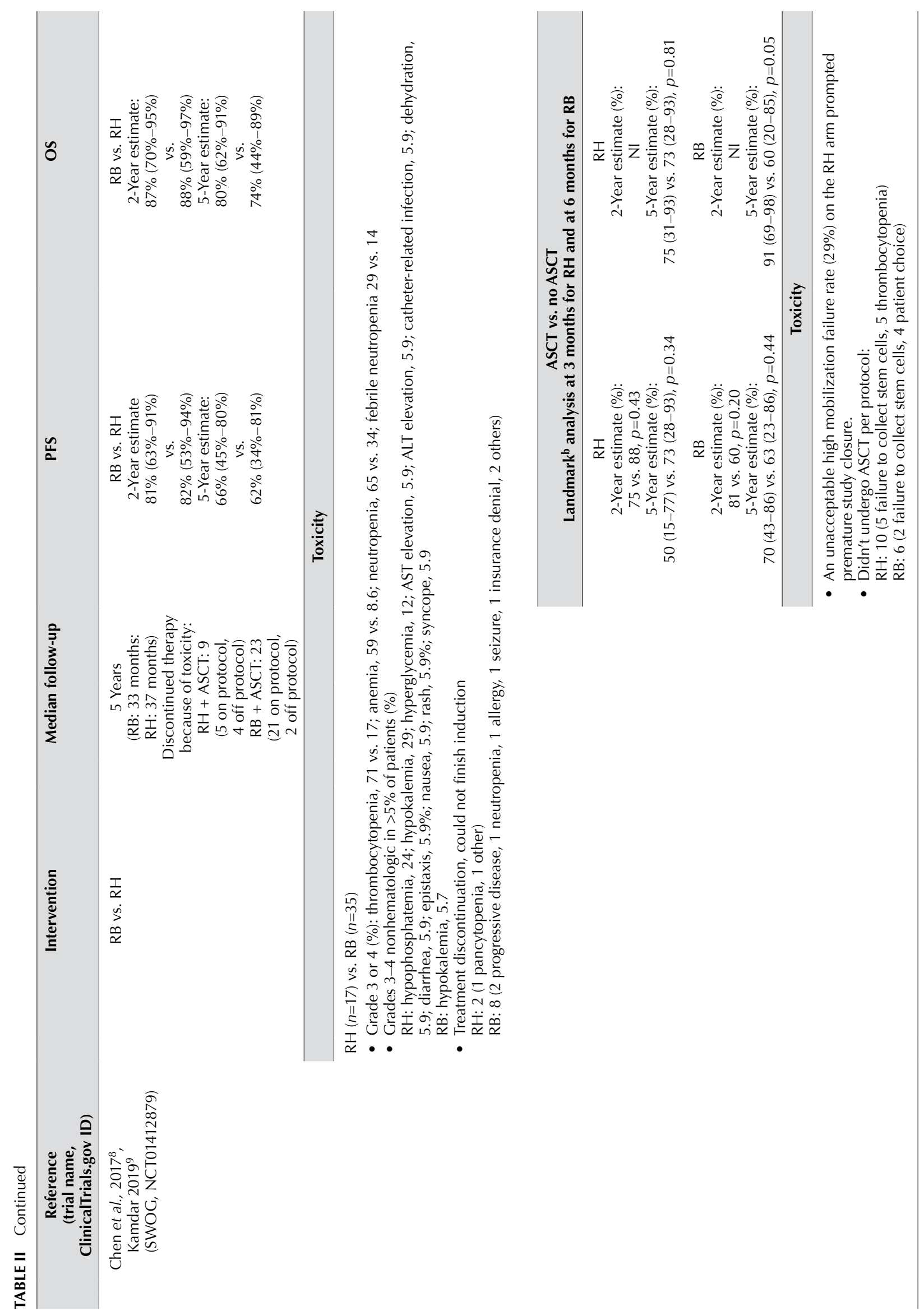




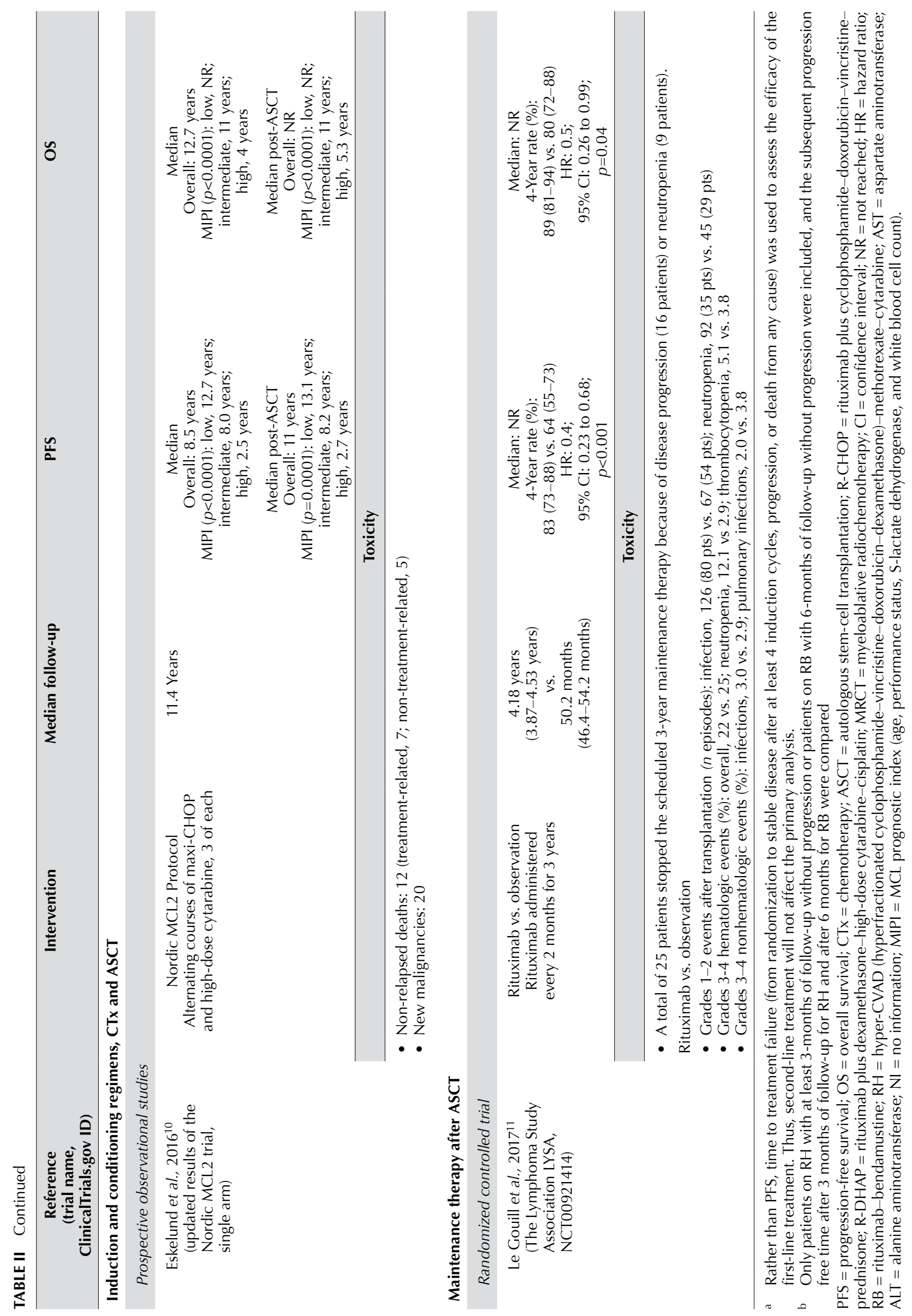




\section{Nordic MCL2}

One single-arm phase II multicentre study ${ }^{10}$ investigated the efficacy of the MCL2 regimen, which consists of doseintensified induction immunochemotherapy with rituximab plus maxi-CHOP (CHOP alternating with rituximab plus high-dose cytarabine), in the treatment of patients newly diagnosed with MCL (Table II). The study, conducted by the Nordic Lymphoma Group, reported that the use of the MCL2 regimen resulted in a median PFS of 8.5 years and a median OS of 12.7 years. The median post-transplantation PFS was 11 years; the median os was NR. However, the regimen was associated with a continuous pattern of relapse and disease-related mortality.

The evidence from the Nordic MCL2 study was considered to be of very low quality because of the nature of the design (noncomparative).

\section{Addition of ASCT in First-Line Treatment}

The literature review identified no relevant studies evaluating the addition of ASCT compared with no ASCT in first-line therapy that met our inclusion criteria.

\section{Post-Transplant Maintenance}

Only one RCT that investigated the efficacy of posttransplantation maintenance therapy for patients with newly diagnosed MCL was identified ${ }^{11}$. In that randomized phase III trial, 240 patients were treated with 4 courses of DHAP every 21 days (additional rescue induction therapy with 4 courses of R-CHOP was administered to patients with a partial response to immunochemotherapy), followed by R-BEAM (rituximab with BEAM) consolidation therapy before ASCT. After ASCT and up to 3 months later, patients were randomized to receive rituximab (a 3 -year maintenance course administered every 2 months after ASCT) or to undergo observation. At a median follow-up of 4 years, a statistically significant improvement in both PFS and os was evident for patients treated with rituximab maintenance compared with those in the observation group (PFS: $83 \%$ vs. $64 \%$; HR: $0.4 ; 95 \%$ CI: 0.23 to $0.68 ; p<0.001$; Os: $89 \%$ vs. $80 \%$; HR: $0.5 ; 95 \%$ CI: 0.26 to $0.99 ; p=0.04$ ). No late effect of rituximab was reported in either arm. After randomization, 16 patients in the rituximab arm experienced disease progression and 13 died; in the observation arm, 37 patients experienced disease progression and 24 died. The major cause of death in each arm was lymphoma: 8 patients in the rituximab arm and 16 in the observation arm (Table II).

The quality of the evidence in this trial was considered high: subjects were adequately randomized, resulting in comparable study groups; participants were treated according to intended interventions and were followed for an extensive period of time, with few lost to follow up; and data were analyzed in accordance with a pre-specified plan (see supplemental Appendix 2 for details).

\section{DISCUSSION}

The present guidance document reviewed the evidence with respect to the best practices for first-line therapy, conditioning regimens, timing of ASCT, and maintenance therapy for patients with MCL. Management of relapsed or refractory ASCT was felt to be outside the scope of the document.
Historically, clinical research into MCL has been challenging because of a low disease incidence. Because of heterogeneity of data and available studies, practice has varied significantly-provincially, nationally, and internationally. Few large prospective RCTs have been conducted in this patient population because of disease rarity. The present work was undertaken in an attempt to standardize practice across the province of Ontario.

Upfront ASCT after induction therapy for MCL is now considered the standard of care in eligible, fit patients, but few modern studies have explored that approach. However, Dreyling et al. ${ }^{3}$ demonstrated that ASCT in first remission is significantly associated with prolonged PFS in MCL, reaching a median of 39 months for patients who underwent transplantation compared with 17 months for those who received interferon alfa instead.

The incorporation of cytarabine into induction regimens before consolidative ASCT is now considered the standard of care for patients with transplantation-eligible disease. The European MCL Network trial is the first randomized trial to demonstrate the beneficial effect of alternating cycles of R-CHOP and R-DHAP as first-line treatment in patients newly diagnosed with ASCT-eligible MCL. Compared with patients treated with R-CHOP $(85 \%)$, those treated with the cytarabine-containing conditioning regimen (84\%) experienced significantly longer PFS (median: 9.1 years vs. 4.3 years; 5 -year rate: $65 \%$ vs. $44 \%$; HR: 0.55 ; $95 \%$ CI: 0.42 to $0.71 ; p<0.0001)^{7}$. The trial demonstrated a significantly greater PFS and provided strong evidence that cytarabine should be incorporated into induction regimens for MCL before consolidative ASCT.

The Working Group found little evidence to support R-hyperCVAD as an initial induction regimen for MCL before ASCT. The S1106 trial aimed to select an induction regimen followed by ASCT consolidation as a platform for development in future trials, comparing RH with RB followed by ASCT in patients newly diagnosed with stage IV MCL. The trial was closed early because of significant toxicities and an unacceptably high rate of stem-cell mobilization failure (29\%) in patients treated with the RH regimen. As a result, $\mathrm{RH}$ was not believed to be a good initial induction regimen for fit patients with transplantation-eligible MCL.

A lack of prospective comparative data meant that the identification of an optimal conditioning regimen for MCL through this systematic review could not be achieved. In the absence of such data, a definitive standard regimen cannot be recommended, and local approaches such as the BEAM, $B E A C$, and TBI-based regimens are all considered reasonable.

With respect to maintenance therapy after consolidative ASCT, one randomized trial was identified that supported the use of maintenance rituximab for patients with newly diagnosed MCL who had undergone ASCT. Compared with post-transplantation observation, the 18 doses of rituximab administered over a 3 -year course of therapy (every 2 months after ASCT) were associated with significantly prolonged PFS and OS. In Ontario, public reimbursement of rituximab as maintenance therapy covers 8 doses. Exploration into expanding the existing maintenance rituximab schedule to 18 doses (every 2 months for 3 years) should be considered, given that the evidence demonstrates improved PFS and os with that expanded access. 


\section{SUMMARY}

Consolidative ASCT in MCL continues to be the standard of care in fit patients with transplantation-eligible disease. A cytarabine-containing induction regimen is considered the standard of care before ASCT. The RH regimen should be avoided as initial treatment given its high toxicity rates and high rate of stem-cell mobilization failure compared with other lines of induction chemotherapy. Maintenance rituximab after ASCT is supported by the current evidence.

Future prospective trials in MCL could explore ideal conditioning regimens in this population and the effect of various induction regimens on stem-cell mobilization yields.

\section{REVIEW PROCESS}

The health research methodologist (NPV) wrote the initial recommendations and qualifying statements pertaining to the management of patients newly diagnosed with ASCT-eligible MCL. The guidance document was circulated to the members of the Mantle Cell Lymphoma Working Group and discussed during a teleconference, after which the draft recommendations were generated. The ensuing guidance document was reviewed by the Report Approval Panel of the Program in Evidence-Based Care (PEBC) - the Scientific Director, the PEBC Assistant Director, and two health research methodologists - to ensure that guideline development was methodologically rigorous and that the evidence-based recommendations are indeed supported by the evidence in a transparent way. The refined guidance document was then presented to the Ontario Health (Cancer Care Ontario) Stem Cell Transplant Advisory Committee to ensure clinical relevance and the utility of the recommendations, and to obtain final approval.

\section{PRACTICE GUIDELINE}

Collectively, evidence from a systematic review of the primary literature, consensus of expert opinion, feedback obtained through the review process, and final approval given by the Ontario Health (Cancer Care Ontario) Stem Cell Transplant Advisory Committee form the basis of this guideline, completed in June 2020.

\section{Target Population}

The target population for this guideline is patients newly diagnosed with ASCT-eligible MCL.

\section{Recommendation 1}

Alternating cycles of R-CHOP/R-DHAP is the recommended first-line treatment before ASCT for symptomatic patients newly diagnosed with MCL.

QualifyingStatement: Alternating cycles of R-CHOP/R-DHAP is the only regimen supported by the evidence. Alternative regimens have not been evaluated in prospective RCTs published to date; thus, uncertainty remains with respect to the clinical benefits and risks of alternative regimens.

\section{Recommendation 2}

Rituximab plus hyperCVAD, alternating with methotrexate and cytarabine is not recommended for the treatment of patients newly diagnosed with MCL.

\section{Recommendation 3}

The BEAM, BEAC, and TBI-based regimens are all reasonable conditioning regimen options for patients with MCL who have responded to first-line therapy and who are undergoing ASCT.

\section{Recommendation 4}

Maintenance therapy with rituximab is recommended for patients with newly diagnosed MCL who have undergone ASCT.

Qualifying Statement: The evidence is insufficient to support or refute the optimal rituximab maintenance schedule. The evidence supports 18 doses of rituximab administered over 3 years. In Ontario, rituximab is funded up to a maximum of 8 doses over 2 years.

\section{IMPLEMENTATION CONSIDERATIONS}

Funding for longer a maintenance regimen should be considered based on the existing evidence. In Ontario, public reimbursement for rituximab as maintenance therapy in previously untreated patients with MCL is 8 doses, but evidence shows that the extended regimen (18 doses over 3 years of maintenance) should be considered.

The use of DHAP in transplantation-eligible patients with MCL could result in an increased need for inpatient chemotherapy resources. Use of carmustine in high-dose chemotherapy regimens before ASCT might result in increased transplantation-related costs.

\section{ACKNOWLEDGMENTS}

The members of the Working Group thank the following individuals for their assistance in developing this guideline: Judy Brown, Sheila McNair, Jonathan Sussman, and Emily Vella for providing feedback on draft versions; the members of the Ontario Health (Cancer Care Ontario) Stem Cell Transplant Advisory Committee for reviewing and formally approving the final version of the document; Faith Maelzer for conducting a data audit; and Sara Miller for copyediting.

The PEBC supports the work of guideline development groups who develop various PEBC products. The guideline development groups are composed of clinicians, other health care providers and decision-makers, methodologists, and community representatives from across the province.

The PEBC is a provincial initiative of Ontario Health (Cancer Care Ontario), supported by the Ontario Ministry of Health $(\mathrm{OMH})$. All work produced by the PEBC is editorially independent from the $\mathrm{OMH}$.

\section{CONFLICT OF INTEREST DISCLOSURES}

We have read and understood Current Oncology's policy on disclosing conflicts of interest, and we declare the following interests: AA has received travel and accommodation support from Janssen Inc. and funding from Pharmacyclics LLC for a trial in which he was the principal investigator. SB has received consultant fees from Janssen Inc., Celgene, Novartis, and Lundbeck. GF has received consultant fees from Janssen Inc., AstraZeneca, and AbbVie, and research funding from Janssen and AbbVie. MC has received funding from Roche and Celgene for a trial in which he was coinvestigator. The remaining authors have no conflicts to disclose.

The conflicts of interest as declared did not disqualify any individual from performing their designated role in the development of this guideline. 


\section{AUTHOR AFFILIATIONS}

*Division of Medical Oncology and Hematology, Princess Margaret Cancer Centre, Toronto, ON; ${ }^{\dagger}$ Program in Evidence-Based Care, Ontario Health (Cancer Care Ontario), and Department of Oncology, McMaster University, Hamilton, ON; ${ }^{\ddagger}$ Division of Hematology, The Ottawa Hospital, Ottawa, ON; ${ }^{\S}$ Malignant Hematology and Stem Cell Transplantation, The Ottawa Hospital, Ottawa, ON; "Division of Medical Oncology and Hematology, Odette Cancer Centre, Sunnybrook Health Sciences Centre, Toronto, ON; ${ }^{\# D i-}$ vision of Malignant Hematology, Juravinski Cancer Centre, and Department of Oncology, McMaster University, Hamilton, ON; ${ }^{* *}$ Patient representative, ON.

\section{REFERENCES}

1. Chen YB, Lane AA, Logan B, et al. Impact of conditioning regimen on outcomes for patients with lymphoma undergoing high-dose therapy with autologous hematopoietic cell transplantation. Biol Blood Marrow Transplant 2015;21:1046-53.

2. Gerson JN, Handorf E, Villa D, et al. Survival outcomes of younger patients with mantle cell lymphoma treated in the rituximab era. J Clin Oncol 2019;37:471-80.

3. Dreyling M, Lenz G, Hoster E, et al. Early consolidation by myeloablative radiochemotherapy followed by autologous stem cell transplantation in first remission significantly prolongs progression-free survival in mantle-cell lymphoma: results of a prospective randomized trial of the European MCL Network. Blood 2005;105:2677-84.

4. U.K. National Institute for Health and Care Excellence (NICE).
Non-Hodgkin's Lymphoma: Diagnosis and Management. London, U.K.: NICE; 2016.

5. Sterne JAC, Savovic J, Page MJ, et al. RoB 2: a revised tool for assessing risk of bias in randomised trials. BMJ 2019;366:14898.

6. Hilal T, Wang Z, Almader-Douglas D, Rosenthal A, Reeder $\mathrm{CB}$, Jain T. Rituximab maintenance therapy for mantle cell lymphoma: a systematic review and meta-analysis. $\mathrm{Am} \mathrm{J}$ Hematol 2018;93:1220-6.

7. Hermine O, Hoster E, Walewski J, et al. Addition of high-dose cytarabine to immunochemotherapy before autologous stem-cell transplantation in patients aged 65 years or younger with mantle cell lymphoma (MCL Younger): a randomised, open-label, phase 3 trial of the European Mantle Cell Lymphoma Network. Lancet 2016;388:565-75.

8. Chen RW, Li H, Bernstein SH, et al. RB but not R-hCVAD is a feasible induction regimen prior to auto-НСT in frontline MCL: results of swOG study S1106. Br J Haematol 2017;176:759-69.

9. Kamdar M, Li H, Chen RW, et al. Five-year outcomes of the S1106 study of R-hyper-CVAD vs R-bendamustine in transplanteligible patients with mantle cell lymphoma. Blood Adv 2019; 3:3132-5.

10. Eskelund CW, Kolstad A, Jerkeman M, et al. 15-year follow-up of the Second Nordic Mantle Cell Lymphoma trial (MCL2): prolonged remissions without survival plateau. BrJ Haematol 2016;175:410-18.

11. Le Gouill S, Thieblemont C, Oberic L, et al. Rituximab after autologous stem-cell transplantation in mantle-cell lymphoma. N Engl J Med 2017;377:1250-60. 